\title{
Constructive Technology
}

\section{Assessment: Systematic Review and Future Study Needs}

Óscar-Iván Rodríguez-Cardoso; Vladimir-Alfonso Ballesteros-

Ballesteros; Manuel-Francisco Romero-Ospina

Citación: Ó.-I. Rodríguez-Cardoso, V.-A. Ballesteros-Ballesteros, M.F. Romero-Ospina, "Constructive Technology Assessment: Systematic Review and Future Study Needs," Revista Facultad de Ingeniería, vol. 30 (55), e12459, 2021.

\section{https://doi.org/10.19053/01211129.v30.n55.2021.12459}

Recibido: Noviembre 21, 2020; Aceptado: Enero 28, 2021;

Publicado: Febrero 06, 2021

Derechos de reproducción: Este es un artículo en acceso abierto distribuido bajo la licencia $\underline{\mathrm{CC} B Y}$

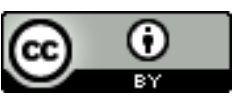

Conflicto de intereses: Los autores declaran no tener conflicto de intereses. 


\title{
Constructive Technology
}

\section{Assessment: Systematic Review and Future Study Needs}

\author{
Óscar-Iván Rodríguez-Cardoso ${ }^{1}$ \\ Vladimir-Alfonso Ballesteros-Ballesteros ${ }^{2}$ \\ Manuel-Francisco Romero-Ospina ${ }^{3}$
}

\begin{abstract}
Engineering, understood as the gathering of scientific and technological knowledge for innovation, creation, advancement and optimization of techniques, as well as a set of useful tools to meet social needs and solve technical problems of both individuals and the community, makes its main actors, engineers, key players in sustainable development and in the creation of alternatives that minimize the negative effects of technology on society. It is in this sense that technology assessment approaches should take importance among those who manage technology development and implementation policies. Generally, the undesirable effects of the intrusion of a new technology are acted upon when they already occur, and technology assessment is intended to anticipate the risk. This paper presents a bibliographic review of technology assessment, its approaches and future study needs. Based on an articulating axis that positions technological change and innovation as an imperative need for social development, an exhaustive review of related articles in specialized databases was carried out. The most important results of this work reveal that the field of technological assessment has been strongly inclined towards the health or sanitary sector; however, research is being developed
\end{abstract}

\footnotetext{
1 M. Sc. Fundación Universitaria Los Libertadores (Bogotá-Distrito Capital, Colombia). oscar.rodriguez@libertadores.edu.co. ORCID: 0000-0003-1203-4999

2 Ph. D. (c) Fundación Universitaria Los Libertadores (Bogotá-Distrito Capital, Colombia). vladimir.ballesteros@libertadores.edu.co. ORCID: 0000-0002-6920-789X

3 M. Sc. Fundación Universitaria Los Libertadores (Bogotá-Distrito Capital, Colombia). mfromeroo@libertadores.edu.co. ORCID: 0000-0002-6457-8827
}

Revista Facultad de Ingeniería (Rev. Fac. Ing.) Vol. 30 (55), e12459. Enero-Marzo 2021. Tunja-Boyacá, 
in central engineering topics such as the development of nanotechnology, robotics, and the handling of big data, where the European model stands out as a reference for technological assessment processes due to its inclusive and democratic nature.

Keywords: constructive technology assessment; responsible innovation; science, technology and society.

\section{Evaluación constructiva de la tecnología: revisión sistemática y necesidades de estudios futuros}

\section{Resumen}

La ingeniería, entendida como la reunión de conocimientos científicos y tecnológicos para la innovación, creación, avance y optimización de técnicas, así como un conjunto de herramientas útiles para suplir las necesidades sociales y resolver problemas técnicos tanto de las personas como de la comunidad, hace a sus principales actores, los ingenieros, piezas clave en el desarrollo sostenible y en la creación de alternativas que minimicen los efectos negativos de la tecnología sobre la sociedad. Es en este sentido que enfoques de evaluación de la tecnología deben tomar importancia entre aquellos que gestionan el desarrollo tecnológico y las políticas de implementación. Generalmente, se actúa frente a los efectos indeseables de la intrusión de una nueva tecnología cuando estos ya ocurren, desde la evaluación tecnológica se pretende anticipar el riesgo. En este documento se presenta una revisión bibliográfica de la evaluación de la tecnología, sus enfoques y necesidades de estudio futuro. A partir de un eje articulador que posiciona el cambio tecnológico y la innovación como una necesidad imperativa para el desarrollo social, se llevó a cabo una revisión exhaustiva de artículos relacionados en bases de datos especializadas. Los resultados más importantes de este trabajo revelan que el campo de la evaluación tecnológica se ha inclinado fuertemente hacia el sector de la salud o sanitario, sin embargo, se desarrollan investigaciones en temas centrales de la ingeniería como el desarrollo de la nanotecnología, la robótica, el manejo de grandes datos donde se destaca el modelo europeo como referente para llevar procesos de evaluación tecnológica por su naturaleza incluyente y democrática. 
Palabras clave: evaluación constructiva de la tecnología; innovación responsable; ciencia, tecnología y sociedad.

\section{Avaliação construtiva da tecnologia: revisão sistemática e necessidades de estudos futuros}

\section{Resumo}

A Engenharia, entendida como o encontro de conhecimentos científicos e tecnológicos para a inovação, criação, avanço e otimização de técnicas, bem como um conjunto de ferramentas úteis para atender às necessidades sociais e solucionar problemas técnicos das pessoas e da comunidade, tem como principal atores, engenheiros, protagonistas do desenvolvimento sustentável e da criação de alternativas que minimizem os efeitos negativos da tecnologia na sociedade. É nesse sentido que as abordagens de avaliação de tecnologia devem ganhar importância entre aqueles que gerenciam o desenvolvimento de tecnologia e as políticas de implementação. Geralmente, ações são tomadas contra os efeitos indesejáveis da intrusão de uma nova tecnologia quando estes já ocorrem, a partir da avaliação tecnológica pretende-se antecipar o risco. Este documento apresenta uma revisão bibliográfica da avaliação de tecnologia, suas abordagens e necessidades para estudos futuros. A partir de um eixo articulador que posiciona a mudança tecnológica e a inovação como uma necessidade imperiosa para o desenvolvimento social, foi realizada uma revisão exaustiva de artigos relacionados em bases de dados especializadas. Os resultados mais importantes deste trabalho revelam que o campo da avaliação tecnológica tem se inclinado fortemente para o setor da saúde ou sanitário, no entanto, pesquisas estão sendo realizadas em temas centrais da engenharia como o desenvolvimento da nanotecnologia, robótica, a gestão de big data onde o modelo europeu destaca-se como referência para a realização de processos de avaliação tecnológica pelo seu caráter inclusivo e democrático.

Palavras-chave: avaliação construtiva de tecnologia; inovação responsável; ciência, tecnologia e sociedade. 


\section{INTRODUCCIÓN}

La Evaluación de la Tecnología (en adelante ET) consiste en un proceso científico, interactivo y comunicativo que tiene por objeto contribuir a la formación de la opinión pública y política sobre los aspectos sociales de la ciencia y la tecnología [1]. La ET se ha definido como una forma de investigación de políticas públicas que examina las consecuencias a corto y largo plazo que pueden asociarse con la vinculación de nuevas tecnologías en distintos escenarios, entre ellos sociales, económicos, éticos y jurídicos [2].

Entre los principales objetivos de la ET están proporcionar información a los encargados de la formulación de políticas sobre alternativas que tengan en cuenta los efectos negativos, así como los positivos de la tecnología sobre la sociedad [3$5]$, explorar los posibles efectos secundarios involuntarios y negativos de la tecnología, elaborar estrategias para hacerles frente y proporcionar asesoramiento en materia de políticas [6-8]. Sin embargo, la ET puede entenderse como un campo de análisis sumamente amplio que abarca también sus conexiones con otras actividades que se llevan a cabo, por ejemplo, tópicos como la difusión de la tecnología y su transferencia, los factores que conducen a una rápida aceptación de la nueva tecnología y sus efectos en la sociedad [2], al mismo tiempo, incorporar evaluaciones ambientales y de sostenibilidad mediante gobiernos reflexivos para contribuir al desarrollo de tecnologías más sostenibles en un marco más amplio de desarrollo tecnológico [6, 9]. La ET es un campo complejo de movimientos distintos estrechamente conectados y entrelazados, tanto en la ciencia como en la política científica y tecnológica que conduce a una participación mucho más profunda de los agentes gubernamentales en el desarrollo de la ciencia y la tecnología; es decir, un desafío fundamental para la ET es abarcar, de manera más explícita, las interacciones entre la dinámica institucional y el desarrollo tecnológico [10]. La ET es un tema vigente en la comunidad científica internacional, por citar algunos ejemplos, el trabajo de Raymond en los países bajos (Netherlands) que empieza en 2010 con su tesis doctoral [31] y continua a la fecha en el campo de la evaluación constructiva de la nanotecnología y sus posibles efectos en la sociedad, o la tesis doctoral de Moretto [32] desarrollada en Portugal en 2017, que analiza mediante 
enfoques constructivos de ET el dilema de la promoción y el control sobre las demandas sociales que se han abierto sin precedentes con el auge de los medios sociales, los grandes datos y su velocidad de transferencia, combinados con el uso generalizado de la tecnología móvil, que requieren nuevas formas para integrarse en el sistema digital emergente, que además vincula nuevos actores de la sociedad que se han convertido en innovadores, y que inevitablemente tienen que formar parte de la cadena de valor y la toma de decisiones. En 2017, se presenta en Alemania una primera ET en temas de Robótica de servicios y trabajo humano con enfoque en la sustitución y la cooperación [34]. En Suiza se realizó en 2018 un estudio para un futuro sostenible para la industria europea del cemento y el hormigón desde la ET para la plena decarbonización de la industria en 2050 [33], allí plantearon múltiples escenarios donde se hace una proyección para la industria de la construcción en Europa que permita la sostenibilidad desde múltiples miradas de los actores implicados. O en la evaluación de la tecnología para la salud por ejemplo para la generación de políticas para las terapias incluidas en el tratamiento del COVID-19 [35].

\section{Metodología}

El presente artículo de revisión es un estudio pormenorizado, selectivo y crítico que integra la información esencial acerca de la evaluación de la tecnología y sus enfoques, así como sus necesidades de estudio futuro. La bibliografía seleccionada fue recuperada de bases de datos especializadas. En total se analizaron cerca de cien artículos que incluían la palabra clave technology assessment, y se clasificaron según sus principales desarrollos para responder las preguntas motivadoras de análisis: ¿cómo ha evolucionado el concepto de evaluación de la tecnología y qué enfoques emergentes han aparecido? $Y$ ¿cuáles son las necesidades de investigación futura en este campo?

\section{Resultados}

La literatura revisada posiciona el surgimiento de la ET durante los años 60; en este periodo se sentaron las bases de la política moderna de ciencia y tecnología, el 
papel cambiante de los gobiernos, las nuevas responsabilidades emergentes de la preocupación pública por las repercusiones del desarrollo y la utilización de las nuevas tecnologías; los encargados de la formulación de políticas iniciaron investigaciones sobre las consecuencias imprevistas y no previstas de las nuevas tecnologías. En ese sentido, se introdujo el análisis y la consideración, hasta entonces descuidados, de las dimensiones no técnicas en el proceso de adopción de decisiones en materia de ciencia y tecnología e instalaron instituciones destinadas específicamente a asesorar a los encargados de la formulación de políticas sobre los avances científicos y las políticas conexas, así como sobre sus repercusiones sociales más amplias, estableciendo así una forma de ET que hoy se considera la ET "clásica" [10].

El surgimiento de la ET puede remontarse a los cambios institucionales en los países occidentales como Estados Unidos, después de la Segunda Guerra Mundial, cuando se introdujeron por primera vez políticas nacionales integrales de investigación científica, así como de apoyo a la ciencia y a la difusión de la tecnología. Posteriormente, su uso se extendió a países europeos como Dinamarca y Holanda [11] aportando nuevos enfoques, más democráticos y participativos. Daddario [12] sugiere en su escrito germinal sobre la ET que, debido a la naturaleza irreversible de muchos cambios tecnológicos y la gran cantidad de consecuencias posibles, la simpatía por asumir riesgos inherentes al avance tecnológico debe ir acompañada de una evaluación más profunda de los posibles impactos negativos y los beneficios sobre la sociedad. De la misma manera, Daddario advirtió que, en esa época, la ET pocas veces se llevaba a cabo y, por lo general, se realizaba mucho después de la incorporación de la tecnología a la sociedad cuando las consecuencias indeseables habían alcanzado proporciones graves.

Entre sus ejemplos, Daddario cita la experiencia del cultivo de pastizales en las Grandes Llanuras y cómo este precipitó las tormentas de polvo y la erosión durante la sequía de la década de 1930; como resultado, los estudios mostraron el camino hacia la acción correctiva de los cortavientos y otras medidas de conservación del suelo que se produjeron después de haber ocasionado dificultades a los agricultores involucrados. La tecnología puede traer beneficios sobre la sociedad, pero también 
puede traer efectos nocivos. El cambio no necesariamente equivale a progreso, sin embargo, es imperativo seguir avanzando en términos tecnológicos y, en este punto, la ET no pretende desanimar al empresario, al inventor, al ingeniero o al educador; de hecho, busca fomentar el mayor grado de imaginación para hacer frente a los problemas de la vida y la existencia, por lo que esta imaginación debe extenderse para incluir políticas de evaluación completa de todas las consecuencias sin el temor de una sociedad reactiva que aproveche los riesgos y los déficits como excusa para el estancamiento. Es decir, la ET tiene por objeto el desarrollo tecnológico bajo la previsión del riesgo y la anticipación de efectos negativos sobre la sociedad [12]. En la Tabla 1, se muestran las siete etapas que debe llevar un proceso de ET según Daddario [12].

Tabla 1. Etapas de la Evaluación de la Tecnología.

\begin{tabular}{|c|l|}
\hline Etapa & \multicolumn{1}{|c|}{ Descripción } \\
\hline 1 & $\begin{array}{l}\text { Identificar todos los posibles impactos de esta tecnología en los sectores natural, social, } \\
\text { económico, jurídico y político. Los efectos directos se separarían de los efectos derivados }\end{array}$ \\
\hline 2 & Establecer relaciones de causa y efecto \\
\hline 3 & Determinar métodos alternativos de aplicación \\
\hline 4 & Determinar tecnologías alternativas para lograr el mismo objetivo \\
\hline 5 & Identificar los impactos de las otras tecnologías \\
\hline 6 & Comparar impactos buenos y malos a partir de mediciones y evidencias significativas \\
\hline 7 & Presentar los resultados \\
\hline
\end{tabular}

En síntesis, el problema de investigación de la ET está orientado a contribuir a soluciones desde la transformación del problema científico en un programa de investigación manejable hasta la retroalimentación de las recomendaciones en el proceso de elaboración de políticas de relevancia y el desarrollo de criterios, que sean, al menos, parcialmente normativos y cargados de valor [13]. No obstante, a pesar de la estructura que plantea la ET, para que tenga relevancia social se espera que sus análisis muestren el impacto social, incluso si este impacto es difícil de medir, ya que normalmente es sólo una fuente de influencia en los procesos polifacéticos e iterativos de toma de decisiones políticas. Uno de sus criterios de calidad más importantes es la transparencia en cuanto a métodos y argumentos. La ET afirma ser neutral e imparcial, sin embargo, ya el diseño de cualquier estudio no suele estar completamente exento de marcos normativos [14]. 
La creciente conciencia social respecto a que la ciencia y la tecnología aportan grandes beneficios, pero al mismo tiempo suelen traer algunos efectos negativos, en forma de consecuencias que se evalúan como indeseables, ha generado un aumento de la ET [15] de la que se ha derivado una familia completa de enfoques de ET, entre ellos están la ET de conciencia, ET estratégica, ET constructiva [11, 16], ET interactiva [17], ET participativa [18] y la ET sanitaria que surgió y se desarrolló en la Oficina de Evaluación de Tecnología (OET) de los Estados Unidos. La Evaluación Constructiva de la Tecnología (ECT) puede verse como un enfoque desarrollado principalmente en los Países Bajos y Dinamarca [19]. La ECT se inspira en la necesidad de resolver la dicotomía entre los beneficios de la tecnología y las consecuencias perjudiciales que vienen con su inclusión y su objetivo es encontrar formas de experimentar con la tecnología en la sociedad con el fin de evitar o conocer los posibles efectos perjudiciales. En general, la literatura señala tres niveles de la ECT. El primero, es la anticipación de los impactos de la tecnología, más que la dependencia de la respuesta a los problemas asociados con el desarrollo; el segundo es el aprendizaje profundo, que es necesario para sacar a la superficie y aclarar los valores básicos implícitos en los diseños tecnológicos; y, en tercer lugar, la "reflexividad" de los actores, que supone una mirada sobre los papeles que desempeñan las diversas partes en el proceso de coproducción de la tecnología y sobre las posibles vías para mejorar el proceso de evaluación de la tecnología [20].

La ECT puede considerarse una práctica de diseño tecnológico, donde la evaluación de los efectos se retroalimenta en el desarrollo de la tecnología de forma iterativa y contiene un elemento de aprendizaje social. Es posible que el gobierno y sus organismos sigan desempeñando un papel importante como iniciadores y reguladores de los procesos de modulación, pero esta función no es exclusiva del gobierno y puede ser asumida por otros agentes sociales [19]. La ECT puede entenderse como permitir a la ET ser parte activa del proceso de construcción, donde una de las principales implicaciones es reconocer que otros actores diferentes a los gubernamentales puedan incidir equitativamente sobre las 
decisiones en cuanto al desarrollo de nuevas tecnologías, por ejemplo, los consumidores y los propios productores [11].

La ECT se centra en la interacción más amplia de la gran variedad de actores, incluida la sociedad, que tienen interés en el desarrollo, el despliegue y la utilización de nuevos campos tecnológicos con base en un enfoque desde la teoría de los sistemas sociotécnicos considerando parte de una red sin fisuras de elementos heterogéneos muy relacionados entre sí, como organizaciones, instituciones, recursos, elementos científicos y la legislación [21]. La ECT se ha desarrollado como una práctica de intervención destinada a alinear las expectativas de los diferentes actores interesados, con el fin de facilitar la generación de nueva tecnología o la innovación de la ya existente [22]. La participación de los diferentes interesados en el proceso de diseño de las tecnologías emergentes debería dar lugar a la aceptación de nuevas tecnologías que se adapten mejor a las necesidades y expectativas de la sociedad [19, 23]. Es un enfoque de previsión donde se examinan múltiples futuros, que valora la interacción de los encargados de adoptar decisiones y las instituciones que darán forma al desarrollo y la adaptación de las nuevas tecnologías [24].

Las nuevas tecnologías no sólo producen nuevos riesgos y beneficios, sino que también alteran el orden simbólico o moral; esos cambios son menos tangibles, pero profundos, y requieren más atención y reflexión. Por lo tanto, la reflexión sobre la investigación y la innovación debería incorporar los ideales normativos, y la ética como un componente de la ECT, para abordar las prácticas de constitución de sujetos, no sólo en términos de cómo los seres humanos son formados por las tecnologías, sino también en términos de la responsabilidad moral que las personas tienen de formar activamente sus vidas con el acompañamiento con estas nuevas tecnologías [25]. Para comprender cómo se produce el desarrollo tecnológico en un sistema, la ECT recomienda un enfoque sociotécnico existente o en evolución sin descuidar la dinámica de múltiples factores [26]. El fenómeno como tal, de los caminos sociotécnicos emergentes y estabilizadores, está ahora ampliamente reconocido. Los actores se anticipan a esos caminos y buscan el que podría 
pensarse como mejor camino, por ejemplo, en la lucha por una norma de la industria o un diseño dominante [27].

Entre los principales hallazgos de esta revisión, se encontraron varios estudios que evidencian procesos de ET en la actualidad. Por ejemplo, la captura y almacenamiento de carbono es la única tecnología disponible para mitigar las emisiones de gases de efecto invernadero a gran escala de los sectores energético e industrial basados en combustibles fósiles en un futuro próximo; sin embargo, la mayoría de los sistemas de almacenamiento de $\mathrm{CO}_{2}$ se encuentran en una fase temprana de desarrollo tecnológico y están aún lejos de la comercialización a gran escala debido a los costos altos, la elevada penalización energética, la seguridad y la confiabilidad y, con mayor acento, las incertidumbres políticas [28].

También se identifica, en la literatura científica, que la ECT se ha convertido en una herramienta política estándar para informar a los responsables de la toma de decisiones que deben gestionar la entrada y el uso de productos farmacéuticos, dispositivos médicos y otras tecnologías (incluidas las intervenciones complejas) dentro de los sistemas sanitarios, por ejemplo, a través del reembolso y la fijación de precios. A pesar de la creciente atención prestada a las actividades de ECT, en los últimos años no ha habido ningún intento de sintetizar exhaustivamente las buenas prácticas o las nuevas buenas prácticas para apoyar la toma de decisiones basadas en la población [29].

Cabe decir que la ET para la salud o sanitaria es el enfoque de ET con mayor cantidad de investigaciones publicadas en los últimos cinco años, desde nuevos enfoques para su definición [36], pasando por revisiones hechas en países de Latinoamérica como Brasil [37], o en Europa [38], para el tratamiento de enfermedades como la diabetes [39] o el cáncer de tiroides [42] en el planteamiento de criterios [40], o en la creación de políticas en países como Tailandia [41].

\section{Discusión Y CONCLUSIONES}

Con plena voluntad política, las comunidades de ET deberían plantearse el reto, por un lado, de ganar la confianza y el apoyo activo de los actores políticos comprometidos con los mismos ideales de democracia y elaboración de políticas 
para el desarrollo tecnológico basadas en el conocimiento. Por otro lado, las comunidades de ET también deberán distinguir a sus partidarios y adversarios y, en consecuencia, reforzar su poder de influencia en la elaboración de políticas. En una época de incertidumbre política y ambigüedad epistémica, las comunidades de ET pueden convertirse en un bastión de la política democrática que den paso a una innovación responsable [30].

Se destaca un enfoque ET acorde a los planteamientos europeos de la ECT donde las políticas para la innovación reflejan un interés por integrar las inquietudes de la sociedad en los procesos de innovación. Se puede tomar como ejemplo el contexto europeo donde se cree en las dinámicas de innovación más inclusivas debido a la experiencia que les trajo el fracaso regulador ligado al desarrollo y comercialización de la biotecnología agroalimentario, donde ha primado la controversia y discordia social acerca de la verdadera evaluación de los riesgos y beneficios de los organismos modificados genéticamente (OMG) aplicados a la agricultura.

Después de esta revisión se pretende advertir que el rol del ingeniero debe considerar el desarrollo tecnológico, no solo desde el ámbito científico sino también debe entender el mundo desde los efectos que tienen sus creaciones sobre la sociedad, las propiedades de la tecnología, sus impactos futuros (incluida la distribución de riesgos y beneficios) se configuran en las interacciones entre los agentes sociales. La conformación de la tecnología, de sus propiedades e impactos se extiende más allá de la etapa de desarrollo hasta la de aplicación, adopción y uso más amplio, por tanto, la composición compleja dada por la interacción de los agentes sociales que se integran cambia de una fase del proceso a la otra, pero cada interacción importante deja sus huellas en la tecnología y en el entorno social. La tecnología y las condiciones sociales coevolucionan en el mismo movimiento y se producen continuamente evaluaciones de diversos tipos. El desafío es prejuzgar estas evaluaciones en la dirección correcta - como parte de un proceso de aprendizaje abierto sobre cuál podría ser la dirección "correcta". 


\section{CONTRIBUCIÓN DE LOS AUTORES}

Óscar-Iván Rodríguez-Cardoso: Conceptualización, Investigación, Metodología, Escritura - borrador original, Escritura - revisión y edición.

Vladimir-Alfonso Ballesteros-Ballesteros: Conceptualización, Investigación, Metodología, Escritura - borrador original, Escritura - revisión y edición.

Manuel-Francisco Romero-Ospina: Conceptualización, Investigación, Metodología, Escritura - borrador original, Escritura - revisión y edición.

\section{REFERENCIAS}

[1] M. Decker, M. Ladikas, S. Stephan, F. Wütscher. Bridges between science, society and policy: technology assessment-methods and impacts, Springer, 2004. https://doi.org/10.1007/978-3-662-06171-8

[2] D. Banta, "What is technology assessment?," International Journal of Technology Assessment in Health Care, vol. 25, no. S1, pp. 7-9, 2009. https://doi.org/10.1017/S0266462309090333

[3] J. F. Coates, "Technology assessment: The benefits... the costs... the consequences," IEEE Engineering Management Review, vol. 2, no. 2, pp. 41-47, 1974. https://doi.org/10.1109/EMR.1974.4306353

[4] S. R. Arnstein, "Technology assessment: Opportunities and obstacles," IEEE Transactions on Systems, Man, and Cybernetics, vol. 7, no. 8, pp. 571-582, 1977. https://doi.org/10.1109/TSMC.1977.4309782

[5] A. H. Teich, Technology and man's future, New York: St. Martin's Press, 1977

[6] A. Grunwald, "Sustainability assessment of technologies - an integrative approach," in Sustainable Development - Energy, Engineering and Technologies - Manufacturing and Environment, InTech, 2012, pp. 35-62

[7] A. Grunwald, "Technology Assessment and Design for Values," in Handbook of Ethics, Values, and Technological Design. Sources, Theory, Values and Application Domains, Netherlands: Springer, pp. 6786, 2015. https://doi.org/10.1007/978-94-007-6994-6 4-1

[8] A. Grunwald, "Diverging pathways to overcoming the environmental crisis: A critique of eco-modernism from a technology assessment perspective," Journal of Cleaner Production, vol. 197, pp. 1854-1862, 2018. https://doi.org/10.1016/i.jclepro.2016.07.212

[9] A. Grunwald, C. Rösch, "Sustainability assessment of energy technologies: Towards an integrative framework," Energy, sustainability and society, vol. 1, e3, 2011. https://doi.org/10.1186/2192-0567-1-3

[10] B. Truffer, J. Schippl, T. Fleischer, "Decentering technology in technology assessment: Prospects for sociotechnical transitions in electric mobility in Germany," Technological Forecasting and Social Change, vol. 122, pp. 34-48, 2017. https://doi.org/10.1016/j.techfore.2017.04.020

[11] A. Rip, "Contributions from social studies of science and constructive technology assessment," On science and precaution in the management of technological risk, vol. 2, pp. 94-122, 2002

[12] E. Q. Daddario, "Technology Assessment," in Statement of Emilio Q. Daddario, Chairman, Subcommittee on Science, Research, and Development of the Committee on Science and Astronautics, US House of Representatives, Ninetieth Congress, First Session (Vol. 2). US Government Printing Office, 1967

Revista Facultad de Ingeniería (Rev. Fac. Ing.) Vol. 30 (55), e12459. Enero-Marzo 2021. Tunja-Boyacá, Colombia. L-ISSN: 0121-1129, e-ISSN: 2357-5328.

DOI: https://doi.org/10.19053/01211129.v30.n55.2021.12459 
Óscar-Iván Rodríguez-Cardoso; Vladimir-Alfonso Ballesteros-Ballesteros; Manuel-Francisco Romero-Ospina

[13] M. Decker, A. Grunwald, "Rational Technology Assessment as Interdisciplinary Research," in Interdisciplinarity in Technology Assessment. Implementation and its Chances and Limits, Berlin, pp 3360, 2001. https://doi.org/10.1007/978-3-662-04371-4 4

[14] A. Grunwald, "Technology Assessment: Concepts and Methods," in Philosophy of Technology and Engineering Sciences, 2009, pp. 1103-1146. https://doi.org/10.1016/B978-0-444-51667-1.50044-6

[15] H. Van Lente, T. Swierstra, P. B. Joly. "Responsible innovation as a critique of technology assessment," Journal of Responsible Innovation, vol. 4, no. 2, pp. 254-261, 2017. https://doi.org/10.1080/23299460.2017.1326261

[16] R. Smits, J. Leyten. P. Den Hertog, "Technology Assessment and Technology Policy in Europe: New Concepts, New Goals, New Infrastructures," Policy Sciences, vol. 28, pp. 271-299, 1995. https://doi.org/10.1007/BF01000290

[17] J. Grin, H. Van der Graaf, "Technology Assessment as Learning," Science, Technology and Human Values, vol. 21, pp. 72-99, 1996. https://doi.org/10.1177/016224399602100104

[18] T. Cronberg, "Technology Assessment in the Danish Socio-Political Context", Assessment Texts No. 9, The Unit of Technology Assessment, Technical University of Denmark, Copenhagen, 2014. https://doi.org/10.1504/IJTM.1996.025456

[19] J. Schot, A. Rip. "The past and future of constructive technology assessment," Technological forecasting and social change, vol. 54, no. 2-3, pp. 251-268, 1997. https://doi.org/10.1016/s0040-1625(96)00180-1

[20] A. Genus, A. M. Coles. "On constructive technology assessment and limitations on public participation in technology assessment," Technology Analysis \& Strategic Management, vol. 17, no. 4, pp. 433-443, 2005. https://doi.org/10.1080/09537320500357251

[21] D. K. R. Robinson, "Constructive technology assessment of emerging nanotechnologies: experiments in interactions," Doctoral Thesis, University of Twente, United Kingdom, 2010

[22] T. Versteeg, M.J. Baumann, M. Weil, A. B. Moniz, "Exploring emerging battery technology for gridconnected energy storage with Constructive Technology Assessment," Technological Forecasting and Social Change, vol. 115, pp. 99-110, 2016. https://doi.org/10.1016/.techfore.2016.09.024

[23] H. Te Kulve, K. Konrad, "The demand side of innovation governance: Demand articulation processes in the case of nano-based sensor technologies," in Bowman D, Stokes E and Rip A (eds). Embedding and Governing New Technologies: A Regulatory, Ethical \& Societal Perspective, Singapore: Pan Stanford, 2017. https://doi.org/10.1201/9781315379593-8

[24] D. Schepis, S. Purchase, N. Ellis. "Understanding Constructive Technology Assessments from an IMP Perspective: The Case of Autonomous Vehicles," in 35th IMP Conference, Paris, 2019, pp. 1-8

[25] A. H. Kiran, N. Oudshoorn, P. P. Verbeek, "Beyond checklists: toward an ethical-constructive technology assessment," Journal of responsible innovation, vol. 2, no. 1, pp. 5-19, 2015. https://doi.org/10.1080/23299460.2014.992769

[26] A. Parandian, A. Rip, "Scenarios to explore the futures of the emerging technology of organic and large area electronics," European journal of futures research, vol. 1, no. 1, pp. 9, 2013. https://doi.org/10.1007/s40309-013-0009-2

[27] K. Konrad, A. Rip, V. C. S. Greiving-Stimberg, "Constructive Technology Assessment-STS for and with technology actors," EASST Review, vol. 36, no. 3, 2017

[28] Z. Zhang, D. Huisingh. "Carbon dioxide storage schemes: technology, assessment and deployment," Journal of Cleaner Production, vol. 142, pp. 1055-1064, 2017. https://doi.org/10.1016/i.jclepro.2016.06.199

Revista Facultad de Ingeniería (Rev. Fac. Ing.) Vol. 30 (55), e12459. Enero-Marzo 2021. Tunja-Boyacá, Colombia. L-ISSN: 0121-1129, e-ISSN: 2357-5328.

DOI: https://doi.org/10.19053/01211129.v30.n55.2021.12459 
Constructive Technology Assessment: Systematic Review and Future Study Needs

[29] F. B. Kristensen, D. Husereau, M. Huić, M. Drummond, M.L. Berger, K. Bond, A. Wailoo. "Identifying the need for good practices in health technology assessment: Summary of the ISPOR HTA Council Working Group Report on Good Practices in HTA," Value in Health, vol. 22, no. 1, pp. 13-20, 2019. https://doi.org/10.1016/i.jval.2018.08.010

[30] P. Delvenne, C. Parotte. "Breaking the myth of neutrality: Technology Assessment has politics, Technology Assessment as politics," Technological Forecasting and Social Change, vol. 139, pp. 64-72, 2019. https://doi.org/10.1016/..techfore.2018.06.026

[31] D. K. R Robinson, "Constructive technology assessment of emerging nanotechnologies: experiments in interactions", Doctoral Thesis, University of Twente, United Kingdom, 2010

[32] S. Moretto, "Technology Assessment and High-Speed Trains: facing the challenge of emergent digital society", Doctoral Thesis, Universidade Nova, Potugal, 2017

[33] A. Favier, C. De Wolf, K. Scrivener, G. Habert, A sustainable future for the European Cement and Concrete Industry: Technology assessment for full decarbonisation of the industry by 2050, ETH Zurich, 2018. https://doi.org/10.3929/ethz-b-000301843

[34] M. Decker, M. Fischer, I. Ott, "Service Robotics and Human Labor: A first technology assessment of substitution and cooperation," Robotics and Autonomous Systems, vol. 87, pp. 348-354, 2017. https://doi.org/10.1016/.j.robot.2016.09.017

[35] P. K. Lorgelly, A. Adler, "Impact of a global pandemic on health technology assessment," Applied health economics and health policy, vol. 18, pp. 339-343, 2020. https://doi.org/10.1007/s40258-020-00590-9

[36] B. O'Rourke, W. Oortwijn, T. Schuller, "The new definition of health technology assessment: A milestone in international collaboration," International journal of technology assessment in health care, vol. 36, no. 3, pp. 187-190, 2020. https://doi.org/10.1017/S0266462320000215

[37] M. S. Ali, M.Y. Ichihara, L. C. Lopes, G. C. Barbosa, R. Pita, R. P. Carreiro, M. L. Barreto, "Administrative data linkage in Brazil: potentials for health technology assessment," Frontiers in pharmacology, vol. 10, e984, 2019. https://doi.org/10.3389/fphar.2019.00984

[38] M. Blüher, S. J. Saunders, V. Mittard, R. Torrejon Torres, R., J. A. Davis, R. Saunders, "Critical review of European health-economic guidelines for the health technology assessment of medical devices," Frontiers in medicine, vol. 6, e278, 2019. https://doi.org/10.3389/fmed.2019.00278

[39] Health Quality Ontario, Hyperbaric oxygen therapy for the treatment of diabetic foot ulcers: a health technology assessment," Ontario health technology assessment series, vol. 17, no. 5, pp. 1-142, 2017

[40] R. Baltussen, K. Marsh, P. Thokala, V. Diaby, H. Castro, I. Cleemput, M. Garau, G. Iskrov, A. Olyaeemanesh, A. Mirelman, M. Mobinizadeh, A. Morton, M. Tringali, J. Van Til, J. Valentim, M. Wagner, S. Youngkong, V. Zah, A. Toll, M. Jansen, L. Bijlmakers, W. Oortwijn, H. Broekhuizen, "Multicriteria decision analysis to support health technology assessment agencies: benefits, limitations, and the way forward," Value in Health, vol. 22, no. 11, pp. 1283-1288, 2019. https://doi.org/10.1016/j.jval.2019.06.014

[41] S. Tantivess, K. Chalkidou, N. Tritasavit, Y. Teerawattananon, "Health Technology Assessment capacity development in low-and middle-income countries: Experiences from the international units of HITAP and NICE," F1000Research, vol. 6, e2119, 2017. https://doi.org/10.12688/f1000research.13180.1

[42] G. Improta, A. Perrone, M. A. Russo, M. Triassi, "Health technology assessment (HTA) of optoelectronic biosensors for oncology by analytic hierarchy process (AHP) and Likert scale," BMC medical research methodology, vol. 19, no. 1, pp. 1-14, 2019. https://doi.org/10.1186/s12874-019-0775-z

Revista Facultad de Ingeniería (Rev. Fac. Ing.) Vol. 30 (55), e12459. Enero-Marzo 2021. Tunja-Boyacá, Colombia. L-ISSN: 0121-1129, e-ISSN: 2357-5328.

DOI: https://doi.org/10.19053/01211129.v30.n55.2021.12459 